Article

\title{
Grazing and Cutting under Different Nitrogen Rates, Application Methods and Planting Density Strongly Influence Qualitative Traits and Yield of Canola Crop
}

\author{
Sajjad Zaheer 1,2, Muhammad Arif ${ }^{2}$, Kashif Akhtar ${ }^{3}{ }^{(D)}$, Ahmad Khan ${ }^{2}$ (D), Aziz Khan ${ }^{4}$ (D), \\ Shahida Bibi ${ }^{5}$, Mehtab Muhammad Aslam ${ }^{6}$ D, Salman Ali ${ }^{2}$, Fazal Munsif ${ }^{2}$, Fazal Jalal ${ }^{7}$, \\ Noor Ul Ain ${ }^{8}$, Fazal Said ${ }^{7}$, Muhammad Ali Khan ${ }^{6}$, Muhammad Jahangir ${ }^{9}$ and Fan Wei ${ }^{1, *}$ \\ 1 Guangxi Key laboratory of medicinal resources protection and genetic improvement, Guangxi botanical \\ garden of medicinal plants, Nanning 530005, China; Sajjadzaheer15@gmail.com \\ 2 Department of Agronomy, The University of Agriculture Peshawar-Pakistan, Peshawar 25000, Pakistan; \\ marifkhan75@aup.edu.pk (M.A.); ahmad0936@yahoo.com (A.K.); salmankhan@aup.pk (S.A.); \\ munsiffazal@yahoo.com (F.M.) \\ 3 Institute of Nuclear Agriculture Sciences, College of Agriculture and Biotechnology, Zhejiang University, \\ Hangzhou 310058, China; kashif@zju.edu.cn \\ 4 College of Agriculture, Guangxi University, Nanning 530005, China; azizkhanturlandi@gmail.com \\ 5 Department of Weed Science, The University of Agriculture Peshawar-Pakistan, Peshawar 25000, Pakistan; \\ shahida@aup.edu.pk \\ 6 Center for Plant Water-Use and Nutrition Regulation, College of Life Sciences, Joint International Research \\ Laboratory of Water and Nutrient in Cops, Fujian Agriculture and Forestry University, Fuzhou 350002, \\ Fujian, China; mehtabmuhammadaslam@yahoo.com (M.M.A.); malikhan@awkum.edu.pk (M.A.K.) \\ 7 Department of Agriculture, Abdul Wali khan University Mardan, Mardan 23200, Pakistan; \\ fazaljalal@awkum.edu.pk (F.J.); fazal@awkum.edu.pk (F.S.) \\ 8 FAFU and UIUC-SIB joint center for genomics and biotechnology, Fujian Agriculture and Forestry \\ University, Fuzhou 350002, Fujian, China; noorulainali001@gmail.com \\ 9 Department of Horticulture, The University of Agriculture Peshawar-Pakistan, Peshawar 25000, Pakistan; \\ jahangir.hayat44@gmail.com \\ * Correspondence: 15277125531@163.com; Tel.: +86-152-7712-5531
}

Received: 28 January 2020; Accepted: 11 March 2020; Published: 16 March 2020

\begin{abstract}
Canola crop has the potential for both seeds and grazing. Optimal planting density, time of nitrogen $(\mathrm{N})$ fertilizer application and rates are the major aspects for successful qualitative traits and canola yield formation. In this content, optimization of planting density, $\mathrm{N}$ levels and its time of application in dual purpose canola are needed. This study was carried out in RCB design with split pot arrangement having three repeats during winter 2012-2013 and 2013-2014. The study evaluated $\mathrm{N}$ levels (120 and $\left.80 \mathrm{~kg} \mathrm{~N} \mathrm{ha}^{-1}\right)$, cutting treatment, $\mathrm{N}$ application timings and planting density $(20$ and 40 plants $\mathrm{m}^{-2}$ ) effects on qualitative traits and yield of canola. No-cut treatment had $7.02 \%, 2.46 \%$, and $4.26 \%$ higher, glucosinolates, oil, and protein content with $31.3 \%$ and $30.5 \%$ higher biological and grain yield respectively, compared with grazed canola. Compared with no-cut canola, grazed canola resulted in $7.74 \%$ of higher erucic acid. Further, application of $\mathrm{N}$ at $120 \mathrm{~kg} \mathrm{~N} \mathrm{ha}^{-1}$ had $8.81 \%, 5.52 \%$, and $6.06 \%$ higher glucosinolates, percent protein, and seed yield, respectively than $80 \mathrm{~kg} \mathrm{~N} \mathrm{ha}^{-1}$. In-addition, the application of $\mathrm{N}$ into two splits was most beneficial than the rest application timings. Cutting had 15\% reduction in grain yield of canola and fetched additional income of 143.6 USD compared with no-cut. Grazing resulted in a $23 \%$ reduction in grain yield while had additional income of 117.7 USD from fodder yield. Conclusively, the application of $\mathrm{N}$ in two splits at $120 \mathrm{~kg} \mathrm{~N}$ $\mathrm{ha}^{-1}$ combined with 20 plants $\mathrm{m}^{-2}$ is a promising strategy to achieve good qualitative attributes and canola yield under dual purpose system.
\end{abstract}


Keywords: dual purpose canola; nitrogen fertilizer; planting density; oil content; grazing

\section{Introduction}

The domestic production can only meet $29 \%$ of the total edible oil requirements of Pakistan and the remaining $71 \%$ was mad through imports [1]. Like other developing countries, Pakistan is also deficit in edible oil production. According to a study conducted the consumer's demand has steadily increased from 0.3 million tons to 2.764 tons during the last two and half decades. The average yield of canola in Pakistan is $839 \mathrm{~kg} \mathrm{ha}^{-1}$ [2], which is very low compared with other agriculturally advanced countries. The European countries have a yield level of $3500 \mathrm{~kg} \mathrm{ha}^{-1}$; Canada $3200 \mathrm{~kg} \mathrm{ha}^{-1}$; and Australia $2000 \mathrm{~kg} \mathrm{ha}^{-} 1$ for canola crop [3]. To cut down these gaps concrete efforts are needed to increase its local production. Canola is an improved form of conventional rape seed variety developed through genetic engineering having erucic acid less than $2 \%$ and $30 \mu \mathrm{molg}^{-1}$ glucosinolates, which are considered the safe limits for health [4]. As compared to other oil crops, it contains less amount of cholesterol [3]. Seed oil concentration was inversely proportional to seed protein concentration in mustard and canola genotypes. Increase in seed yield increased the oil concentration, but decreased the protein concentration [5].

Optimum amount of nutrients supply at proper time to any crop is important [6]. Canola crop requires a higher amount of nutrients, and available nitrogen $(\mathrm{N})$ compared with cereals [7]. Split application of $\mathrm{N}$ fertilizer has become more popular in terms of high nitrogen use efficiency. An appropriate rate and timing of $\mathrm{N}$ fertilizer application is one of the most important aspects of successful canola production [3]. Canola yield is strongly correlated with biotic and abiotic factors; one of the factors that availability of nutrients especially $\mathrm{N}$ is the key driver for improving root growth, leaf photosynthetic rate, biomass production, and yield [8]. N fertilizer boosts yield improving thousand seed weights, seeds pod ${ }^{-1}$, and pod number plant ${ }^{-1}$ [9]. Dual purpose cropping is the use of crops for fodder purpose at vegetative stage grazed by animals. The regrowth of plants after cutting or grazing strongly relies on the regenerative ability of the species. After being in stress condition, soon after grazing crops $\mathrm{N}$ fertilization is needed for growth improvement, thus $\mathrm{N}$ fertilizer selection is a good choice for growth improvement. Increasing $\mathrm{N}$ level from normal or recommended dozes boosts the overall plant health and seed production [9]. Considering DP canola, $\mathrm{N}$ fertilizer might be increased for better re-growth or regeneration of canola. Moreover, $\mathrm{N}$ is the most volatile and due to high losses, $\mathrm{N}$ efficiency becomes lesser and thereby affects plant normal functioning [10]. Winter or long-season spring canola with proper $\mathrm{N}$ rates can be sown to produce high-quality forage for grazing or fodder for cut and carry and recover from grazing to produce a high grain yield ( $4 \mathrm{t} / \mathrm{ha}$ ) with good oil content $(47 \%)$ [11]. Canola crop has the potential to produce grains and to graze. Although, canola crop is good for forage. Canola grazing is only one of the assortments of choice to the farmers to perk up farm economics and productivity.

However, oil quality is also important, and contains less amount of cholesterol, which is good for human health. Studies regarding $\mathrm{N}$ rate, timing, and planting density under dual purpose canola production are lacking. The objectives of this study were to explore canola qualitative and yield attributes to different $\mathrm{N}$ rate, application, and planting density under dual purpose canola technology. This study also explores the quantitative relationship among $\mathrm{N}$ level, application time and planting density for dual purpose use of canola. Furthermore, the combination of all above discussed factors will make an understanding of the effect of grazing on the yield and quality of canola crop and even development of commercial grazing practices. The tested hypothesis was that $\mathrm{N}$ rate, time of application, and planting density would improve canola qualitative traits and yield under dual purpose technology. 


\section{Materials and Methods}

\subsection{Experimental Site}

The present study was conducted at New Developmental Farm of Agricultural University Peshawar during Rabi 2012-2013. The research farm is located about $300 \mathrm{~m}$ above the sea level, while the site has $34^{\circ} \mathrm{N}$ latitude and $72^{\circ} \mathrm{E}$ longitude. The soil of the site was clay loamy having $\mathrm{pH}$ values ranges between 7.0-7.5. Temperature $\left({ }^{\circ} \mathrm{C}\right)$ and rainfall $(\mathrm{mm})$ during the crop growing season have been shown in Figure 1. Weather data were collected from the meteorological station located near the experimental site.

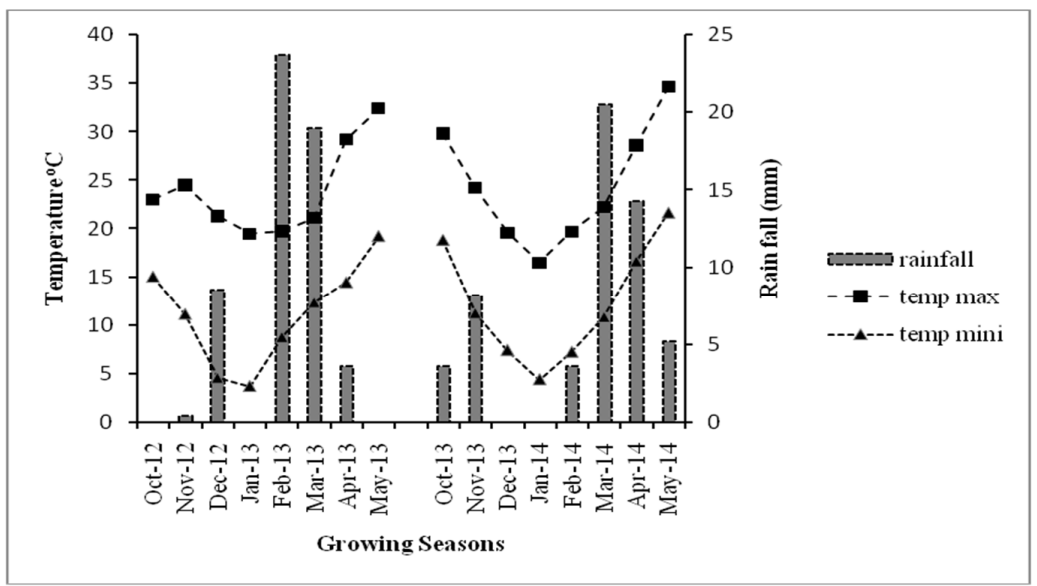

Figure 1. Annual rainfall, maximum and minimum temperature of the experimental site for 2012-2013 and 2013-2014.

\subsection{Treatments and Methods}

The experiment contained two levels (80 and $120 \mathrm{~kg} \mathrm{ha}^{-1}$ ) of nitrogen levels (NL), cuttings (C; (cut, no cut and grazing), application timing (NT; (a) full application at sowing, (b) half dose of N both at sowing and start of rosette stage, (c) one third dose of $\mathrm{N}$ each at sowing, start of rosette stage and

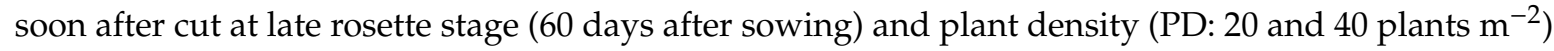
(Table 1). In 2012-2014, the experiment was conducted in RCB design with split plot arrangement having three replications. Cutting and $\mathrm{N}$ levels (urea as a source of nitrogen was applied as top dressed) were assigned to the main plot while $\mathrm{N}$ application timings and planting densities were allotted to sub-plot. Cutting and grazing were done 60 days after sowing. Cutting was done manually by cutting the crop at about $10 \mathrm{~cm}$ above the ground. However, grazing was done through sheep for predetermined time.

Table 1. The detailed presentation of experimental treatments.

\begin{tabular}{|c|c|c|c|}
\hline Main Plot Factors & Treatment Levels & Sub Plot Factors & Treatment Levels \\
\hline Cuttings (C) & $\begin{array}{l}\text { No-cut } \\
\text { (C1) }\end{array}$ & $\begin{array}{l}\text { Nitrogen application } \\
\text { timings (NT) }\end{array}$ & (1) full application at sowing $\left(\mathrm{NT}_{1}\right)$ \\
\hline & Grazing (C3) & & $\begin{array}{l}\text { (2) half dose of } \mathrm{N} \text { both at sowing } \\
\text { and start of rosette stage }\left(\mathrm{NT}_{2}\right) \\
(3) \text { one third dose of } \mathrm{N} \text { each at } \\
\text { sowing, start of rosette stage and } \\
\text { soon after cut at late rosette stage } \\
\text { (60 days after sowing }\left(\mathrm{NT}_{3}\right)\end{array}$ \\
\hline Nitrogen Levels (NL) & $\begin{array}{l}80 \mathrm{~kg} \mathrm{ha}^{-1}\left(\mathrm{NL}_{1}\right) \\
120 \mathrm{~kg} \mathrm{ha}^{-1}\left(\mathrm{NL}_{2}\right)\end{array}$ & Planting density (PD) & $\begin{array}{l}20 \text { plants } \mathrm{m}^{-2}\left(\mathrm{PD}_{1}\right) \\
40 \text { plants } \mathrm{m}^{-2}\left(\mathrm{PD}_{2}\right)\end{array}$ \\
\hline
\end{tabular}

\subsection{Field Preparation and Cultural Practices}

Cultivar Abasin-95 was sown with a uniform seed rate of $8 \mathrm{~kg} \mathrm{ha}^{-1}$. Row to row distance of $50 \mathrm{~cm}$ was maintained with a subplot size of $10.5 \mathrm{~m}^{2}$, having 7 rows, and $3 \mathrm{~m}$ long. Before sowing, a fine 
seedbed was prepared by ploughing the field with cultivator followed by rotavator. A basal dose of phosphorus at $60 \mathrm{~kg} \mathrm{ha}^{-1}$ was applied in the form of single super phosphate. Nitrogenous fertilizer was applied in the form of urea. Weeds were controlled manually by hoeing, when the crop reached $6-8 \mathrm{~cm}$ height. The field was harvested on 10 April each year. All cultural practices were carried out uniformly in all plots.

\subsection{Grazing Management}

Sheep stock was arranged for grazing canola from nearby village. The sheep were allowed to graze a normal canola field about five days before the treatment grazing to acclimatize them to canola/brassica consumption. The sheep were controlled with the help of fences from going to other treatment plots. Animals for grazing were allowed in noon time because of much frost in morning time during grazing period in the month of December in both years.

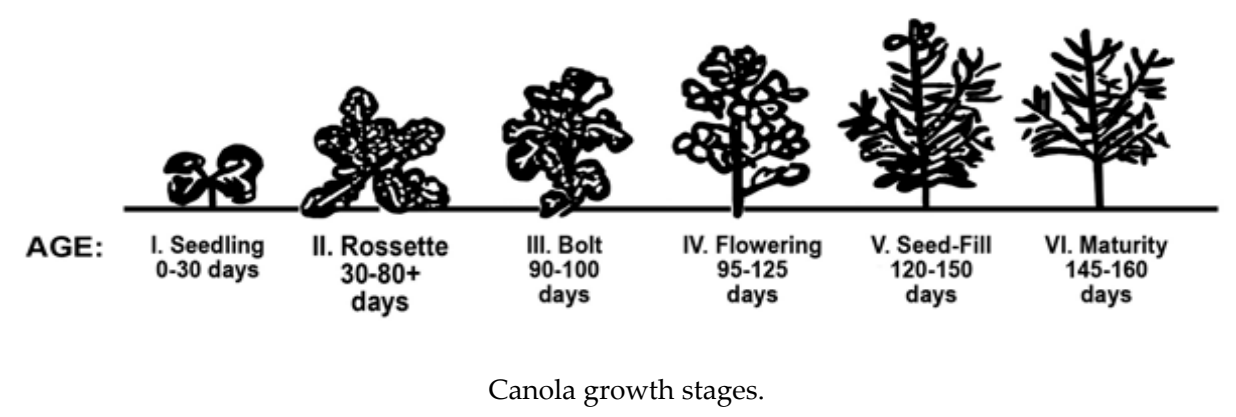

\subsection{Observations}

\subsubsection{Quality Attributes of Canola}

Protein, percent oil content, erucic acid, and glucosinolates were determined by collecting randomly seed samples in each plot and were analyzed by Full Option Science System (FOSS) Routine Near Measurement System (35RP-3752F) TR-3657-C Model 6500, at oilseed laboratory, Nuclear Institute for Food and Agriculture, Peshawar (NIFA). Near infrared reflectance (NIR) spectroscopy is a quick and whole seed analyzing method, which does not require any sample preparation or chemicals [12].

\subsubsection{Canola Yield}

Biomass yield was determined by harvesting of four central rows, dried and weighted. While in order to determine grain yield, bundles from the same central four rows were threshed, seeds were weighed and the data were converted to $\mathrm{kg} \mathrm{ha}^{-1}$ [13].

\subsubsection{Statistical Analysis}

The data were statistically analyzed over years using ANOVA techniques appropriate for RCB design with split plot arrangement using SPSS software (SPSS Inc., Chicago, IL, USA). Means were compared using LSD test at 0.05 level of probability, when the $F$-values were significant [14].

\section{Results}

\subsection{Treatments Interactions}

The significance ANOVA for main factors and interaction is presented in Table 2. The $\mathrm{C} \times \mathrm{NL}$ significantly affected on Glucosinolates, while the rest of treatments interaction were not significant. Oil content were significantly affected by the $\mathrm{C} \times \mathrm{NL}, \mathrm{C} \times \mathrm{NT}$, and NT $\times \mathrm{PD}$ treatments. Protein content was not significant throughout the treatments' interactions. Eurcic acid showed significant differences on $\mathrm{C} \times \mathrm{PD}$ treatment, while biological yield was significant on $\mathrm{C} \times \mathrm{NL}$, and $\mathrm{NT} \times \mathrm{NL}$, and grain yield was on $\mathrm{C} \times \mathrm{NT}$, and $\mathrm{NT} \times \mathrm{PD}$ treatments. 
Table 2. Mean square table for crop yield, qualitative attributes, glucosinulates, and erucic acid for the years 2012-14.

\begin{tabular}{|c|c|c|c|c|c|c|}
\hline SOV & Grain Yield & $\begin{array}{l}\text { Biological } \\
\text { Yield }\end{array}$ & Oil Content & $\begin{array}{l}\text { Protein } \\
\text { Content }\end{array}$ & $\begin{array}{c}\text { Glucosinulates } \\
\text { Content }\end{array}$ & Eurcic Acid \\
\hline Year & $134^{\mathrm{ns}}$ & $7.397 *$ & $1598.49 *$ & $10,560.2$ & $799.26 *$ & 346.56 * \\
\hline Cuttings (C) & $1,482,015^{*}$ & $1.383^{*}$ & $18.93 *$ & $27.7^{\mathrm{ns}}$ & $330.24 *$ & $115.59 *$ \\
\hline N-levels (NL) & $204,857 *$ & $5,140,844^{\mathrm{ns}}$ & 243.63 * & 135.9 & $1547.22 *$ & $22.556^{\mathrm{ns}}$ \\
\hline Planting densities (PD) & $45,182^{\mathrm{ns}}$ & $4,630,281 *$ & $18.84^{*}$ & $1.2^{\mathrm{ns}}$ & $1.23^{\mathrm{ns}}$ & $6.476^{\mathrm{ns}}$ \\
\hline Nitrogen timings (NT) & $30,938^{\mathrm{ns}}$ & $6,378,573 *$ & $23.12 *$ & $1.9^{\mathrm{ns}}$ & $6.18^{\mathrm{ns}}$ & $14.529^{\mathrm{ns}}$ \\
\hline $\mathrm{C} \times \mathrm{NL}$ & ns & * & * & ns & * & ns \\
\hline $\mathrm{C} \times \mathrm{NT}$ & $*$ & ns & * & ns & ns & * \\
\hline $\mathrm{C} \times \mathrm{PD}$ & ns & ns & ns & ns & ns & ns \\
\hline $\mathrm{NL} \times \mathrm{PD}$ & ns & ns & ns & ns & ns & ns \\
\hline $\mathrm{NT} \times \mathrm{NL}$ & ns & $*$ & ns & ns & ns & ns \\
\hline $\mathrm{NT} \times \mathrm{PD}$ & $*$ & ns & $*$ & ns & ns & * \\
\hline $\mathrm{C} \times \mathrm{NT} \times \mathrm{NL}$ & ns & ns & ns & ns & ns & ns \\
\hline $\mathrm{C} \times \mathrm{NT} \times \mathrm{PD}$ & ns & ns & ns & ns & ns & ns \\
\hline $\mathrm{NT} \times \mathrm{NL} \times \mathrm{PD}$ & ns & ns & ns & ns & ns & ns \\
\hline $\mathrm{C} \times \mathrm{NL} \times \mathrm{NT} \times \mathrm{PD}$ & ns & ns & ns & ns & ns & ns \\
\hline
\end{tabular}

Note: $\mathrm{ns}=$ non-significant ${ }^{*}=$ Significant at $5 \%$ level of probability.

\subsection{Crop Yield}

The significance ANOVA for main factors is presented in Table 2. Canola biomass yield was significantly affected by C, PD, and year, whereas NL and NT did not affect biomass yield (Table 3). $\mathrm{C}_{3}$ and $C_{2}$ decreased biological yield by $11.74 \%$ and $31.2 \%$ compared with that of $C_{1}$. Plants grown at $\mathrm{PD}_{2}$ had 5.3\% higher biological yield than $\mathrm{PD}_{1}$. The $\mathrm{C}, \mathrm{NL}$, and NT significantly influenced grain yield of canola, whereas the effect of planting density and year remained unaffected on grain yield (Table 4). The interactions among $C \times N T$ and NT $\times$ PD were significant. Higher grain yield was produced in $C_{1}$ plots, followed by $C_{2}$ plots, whereas lower grain yield resulted in $C_{3}$ plots. Grain yield was higher when $\mathrm{N}$ was applied at $\mathrm{NT}_{2}$ as compared to $\mathrm{NT}_{3}$.

Table 3. Biomass yield $\left(\mathrm{kg} \mathrm{ha}^{-1}\right)$ of canola under cutting treatments, nitrogen levels and application timings under different planting densities.

\begin{tabular}{|c|c|c|c|}
\hline Variables & 2012-2013 & 2013-2014 & Two Years Average \\
\hline \multicolumn{4}{|c|}{ Cutting treatments $(\mathrm{C})$} \\
\hline $\mathrm{C}_{1}$ & 9695 a & 9353 a & $9524 a$ \\
\hline $\mathrm{C}_{2}$ & $7272 b$ & $9774 \mathrm{a}$ & $8523 \mathrm{~b}$ \\
\hline $\mathrm{C}_{3}$ & $6080 c$ & 8432 b & $7256 \mathrm{c}$ \\
\hline $\operatorname{LSD}_{(0.05)}$ & 598 & 631 & 407 \\
\hline \multicolumn{4}{|c|}{ Nitrogen levels (NL) } \\
\hline $\mathrm{NL}_{1}$ & 8099 & 8794 b & 8446 \\
\hline $\mathrm{NL}_{2}$ & 7932 & 9578 a & 8755 \\
\hline Significance level & ns & * & ns \\
\hline \multicolumn{4}{|l|}{$\begin{array}{l}\text { N application timings } \\
\text { (NT) }\end{array}$} \\
\hline $\mathrm{NT}_{1}$ & $7974 \mathrm{ab}$ & 9023 & 8496 \\
\hline $\mathrm{NT}_{2}$ & $7700 \mathrm{~b}$ & 9428 & 8564 \\
\hline $\mathrm{NT}_{3}$ & $8372 \mathrm{a}$ & 9107 & 8739 \\
\hline $\operatorname{LSD}_{(0.05)}$ & 500 & 506 & 352 \\
\hline \multicolumn{4}{|l|}{ Planting density (PD) } \\
\hline $\mathrm{PD}_{1}$ & $7785 \mathrm{~b}$ & $8584 \mathrm{~b}$ & 8454 b \\
\hline $\mathrm{PD}_{2}$ & 8264 a & 9787 a & 8747 a \\
\hline Significance level & * & * & * \\
\hline Year $\left({ }^{*}\right)$ & $8016 \mathrm{~b}$ & 9186 a & \\
\hline
\end{tabular}

Note: Cuttings $(\mathrm{C})$ : no-cut $\left(\mathrm{C}_{1}\right)$, cutting $\left(\mathrm{C}_{2}\right)$, grazing $\left(\mathrm{C}_{3}\right)$; Nitrogen levels $(\mathrm{NL}): 80 \mathrm{~kg} \mathrm{ha}^{-1}\left(\mathrm{NL}_{1}\right), 120 \mathrm{~kg} \mathrm{ha}^{-1}\left(\mathrm{NL}_{2}\right)$; Nitrogen timings: Full application at sowing $\left(\mathrm{NT}_{1}\right)$, half dose of $\mathrm{N}$ both at sowing and start of rosette stage $\left(\mathrm{NT}_{2}\right)$, one third dose of $\mathrm{N}$ each at sowing, start of rosette stage and soon after cut at late rosette stage (60 days after sowing $\left(\mathrm{NT}_{3}\right)$; Planting density (PD): 20 plants $\mathrm{m}^{-2}\left(\mathrm{PD}_{1}\right), 40$ plants $\mathrm{m}^{-2}\left(\mathrm{PD}_{2}\right)$. Means of the same category followed by different letters are significantly different at $5 \%$ level of probability using LSD $_{(0.05)}$ test. ns $=$ non-significant, * = Significant at $5 \%$ level of probability. 
Table 4. Grain yield $\left(\mathrm{kg} \mathrm{ha}^{-1}\right)$ of canola under cutting treatments, nitrogen levels and application timings under different planting densities.

\begin{tabular}{|c|c|c|c|}
\hline Variables & 2012-2013 & 2013-2014 & Two Years Average \\
\hline \multicolumn{4}{|c|}{ Cutting treatments $(\mathrm{C})$} \\
\hline $\mathrm{C}_{1}$ & $1291 \mathrm{a}$ & 1128 a & $1210 \mathrm{a}$ \\
\hline $\mathrm{C}_{2}$ & $983 \mathrm{~b}$ & $1068 \mathrm{~b}$ & $1025 \mathrm{~b}$ \\
\hline $\mathrm{C}_{3}$ & $886 \mathrm{c}$ & $968 c$ & $927 c$ \\
\hline $\operatorname{LSD}_{(0.05)}$ & 72.0 & 54.1 & 42.2 \\
\hline \multicolumn{4}{|c|}{ Nitrogen levels (NL) } \\
\hline $\mathrm{NL}_{1}$ & $1022 \mathrm{~b}$ & $1024 \mathrm{~b}$ & $1023 \mathrm{~b}$ \\
\hline $\mathrm{NL}_{2}$ & $1084 \mathrm{a}$ & $1086 \mathrm{a}$ & $1085 \mathrm{a}$ \\
\hline Significance level & * & * & * \\
\hline \multicolumn{4}{|l|}{$\begin{array}{l}\mathrm{N} \text { application timings } \\
\text { (NT) }\end{array}$} \\
\hline $\mathrm{NT}_{1}$ & $1021 \mathrm{~b}$ & $1054 \mathrm{a}$ & $1045 \mathrm{ab}$ \\
\hline $\mathrm{NT}_{2}$ & $1029 \mathrm{~b}$ & $1058 \mathrm{a}$ & 1078 a \\
\hline $\mathrm{NT}_{3}$ & 1109 a & $1052 \mathrm{a}$ & $1039 \mathrm{~b}$ \\
\hline $\operatorname{LSD}_{(0.05)}$ & 42.5 & 64.6 & 38.3 \\
\hline \multicolumn{4}{|l|}{ Planting density (PD) } \\
\hline $\mathrm{PD}_{1}$ & 1066 & 1057 & 1039 \\
\hline $\mathrm{PD}_{2}$ & 1044 & 1053 & 1068 \\
\hline Significance level & ns & ns & ns \\
\hline Year $(\mathrm{ns})$ & 1053 & 1055 & \\
\hline
\end{tabular}

Note: Cuttings $(\mathrm{C})$ : no-cut $\left(\mathrm{C}_{1}\right)$, cutting $\left(\mathrm{C}_{2}\right)$, grazing $\left(\mathrm{C}_{3}\right)$; Nitrogen levels $(\mathrm{NL}): 80 \mathrm{~kg} \mathrm{ha}^{-1}\left(\mathrm{NL}_{1}\right), 120 \mathrm{~kg} \mathrm{ha}^{-1}\left(\mathrm{NL}_{2}\right)$; Nitrogen timings: Full application at sowing $\left(\mathrm{NT}_{1}\right)$, half dose of $\mathrm{N}$ both at sowing and start of rosette stage $\left(\mathrm{NT}_{2}\right)$, one third dose of $\mathrm{N}$ each at sowing, start of rosette stage and soon after cut at late rosette stage (60 days after sowing $\left(\mathrm{NT}_{3}\right)$; Planting density (PD): 20 plants $\mathrm{m}^{-2}\left(\mathrm{PD}_{1}\right), 40$ plants $\mathrm{m}^{-2}\left(\mathrm{PD}_{2}\right)$. Means of the same category followed by different letters are significantly different at $5 \%$ level of probability using $\operatorname{LSD}_{(0.05)}$ test. ns = non-significant, * = Significant at $5 \%$ level of probability.

\subsection{Quality Parameters of Canola}

\subsubsection{Oil Content (\%) and Protein Content (\%)}

Cuttings, PD, NL, NT, and years significantly affected oil content of canola crop (Table 5). A 1.4\% higher oil content was resulted in $C_{1}$ followed by $C_{2}$. The $C_{3}$ plots substantially reduced oil content. Application of NL1 had $5.07 \%$ of higher oil content compared with $\mathrm{NL}_{2}$. Oil content was higher $\mathrm{NT}_{2}$ followed by $\mathrm{NT}_{3}$ and $\mathrm{NT}_{1}$. Between planting densities, plants under $\mathrm{PD}_{2}$ had higher oil content compared with $P_{1}$. The $C \times N L$ interaction showed an obvious reduction in oil content for $C_{2}$ and $C_{3}$ plots under $\mathrm{NL}_{2}$, whereas the oil content of all cutting treatments remained unchanged under $\mathrm{NL}_{1}$ application (Figure 2A). Interaction between $\mathrm{C} \times \mathrm{NT}$ showed that canola oil content decreased in $\mathrm{C}_{3}$ plots at $\mathrm{NT}_{2}$ than $\mathrm{C}_{1}$ and $\mathrm{C}_{2}$. On other hand, oil content of all cutting treatments remained unchanged under three equal splits application of N (Figure 2B). Interaction between NT and PD indicated that oil content was reduced for $\mathrm{PD}_{1}$ with sole $\mathrm{N}$ at sowing than two or three splits and planting densities (Figure 2C). Cuttings, NL and years had remarkedly effects on grain protein content of canola seeds while the effects of NT and PD were insignificant (Table 5). Higher protein content was noted for cut plots which were statistically at par with $C_{3}$ plots, while $C_{1}$ plots had lower crude protein content in canola seed. The application of $\mathrm{NL}_{2}$ had $5.5 \%$ higher crude protein content compared with that of $\mathrm{NL}_{1}$ (Table 6). 
Table 5. Canola oil content (\%) in response to cutting treatments, nitrogen levels and application timings under varying planting densities.

\begin{tabular}{|c|c|c|c|}
\hline Variables & 2012-2013 & 2013-2014 & Two Years Average \\
\hline \multicolumn{4}{|c|}{ Cutting treatments $(\mathrm{C})$} \\
\hline $\mathrm{C}_{1}$ & $40.78 \mathrm{a}$ & $45.95 \mathrm{a}$ & $43.37 \mathrm{a}$ \\
\hline $\mathrm{C}_{2}$ & $40.03 \mathrm{~b}$ & $45.51 \mathrm{ab}$ & $42.77 \mathrm{~b}$ \\
\hline $\mathrm{C}_{3}$ & $39.51 \mathrm{~b}$ & $45.18 \mathrm{~b}$ & $42.35 \mathrm{c}$ \\
\hline $\operatorname{LSD}_{(0.05)}$ & 0.67 & 0.59 & 0.42 \\
\hline \multicolumn{4}{|c|}{ Nitrogen levels (NL) } \\
\hline $\mathrm{NL}_{1}$ & $41.13 \mathrm{a}$ & $46.66 \mathrm{a}$ & $43.89 \mathrm{a}$ \\
\hline $\mathrm{NL}_{2}$ & $39.09 \mathrm{~b}$ & $44.44 \mathrm{~b}$ & $41.77 \mathrm{~b}$ \\
\hline Significance level & * & * & * \\
\hline \multicolumn{4}{|l|}{$\begin{array}{l}\mathrm{N} \text { application timings } \\
\text { (NT) }\end{array}$} \\
\hline $\mathrm{NT}_{1}$ & $39.81 \mathrm{~b}$ & $45.33 \mathrm{ab}$ & $42.26 \mathrm{~b}$ \\
\hline $\mathrm{NT}_{2}$ & $39.77 \mathrm{~b}$ & $45.09 \mathrm{~b}$ & 43.39 a \\
\hline $\mathrm{NT}_{3}$ & 40.74 a & $46.22 \mathrm{a}$ & $42.83 \mathrm{ab}$ \\
\hline $\operatorname{LSD}_{(0.05)}$ & 0.75 & 0.90 & 0.58 \\
\hline \multicolumn{4}{|l|}{ Planting density (PD) } \\
\hline $\mathrm{PD}_{1}$ & 40.00 & 45.35 & $42.53 \mathrm{~b}$ \\
\hline $\mathrm{PD}_{2}$ & 40.21 & 45.76 & $43.12 \mathrm{a}$ \\
\hline Significance level & * & ns & * \\
\hline Year $(*)$ & $40.11 \mathrm{~b}$ & $45.55 \mathrm{a}$ & \\
\hline
\end{tabular}

Note: Cuttings $(C)$ : no-cut $\left(\mathrm{C}_{1}\right)$, cutting $\left(\mathrm{C}_{2}\right)$, grazing $\left(\mathrm{C}_{3}\right)$; Nitrogen levels $(\mathrm{NL}): 80 \mathrm{~kg} \mathrm{ha}^{-1}\left(\mathrm{NL}_{1}\right), 120 \mathrm{~kg} \mathrm{ha}^{-1}\left(\mathrm{NL}_{2}\right)$; Nitrogen timings: Full application at sowing $\left(\mathrm{NT}_{1}\right)$, half dose of $\mathrm{N}$ both at sowing and start of rosette stage $\left(\mathrm{NT}_{2}\right)$, one third dose of $\mathrm{N}$ each at sowing, start of rosette stage and soon after cut at late rosette stage (60 days after sowing $\left(\mathrm{NT}_{3}\right)$; Planting density (PD): 20 plants $\mathrm{m}^{-2}\left(\mathrm{PD}_{1}\right), 40$ plants $\mathrm{m}^{-2}\left(\mathrm{PD}_{2}\right)$. Means of the same category followed by different letters are significantly different at $5 \%$ level of probability using $\operatorname{LSD}_{(0.05)}$ test. ns = non-significant, * = Significant at $5 \%$ level of probability.

Table 6. Canola protein content (\%) in response to cutting treatments, nitrogen levels and application timings under varying planting densities.

\begin{tabular}{|c|c|c|c|}
\hline Variables & 2012-2013 & 2013-2014 & Two Years Average \\
\hline \multicolumn{4}{|c|}{ Cutting treatments $(\mathrm{C})$} \\
\hline$C_{1}$ & 36.26 & $21.56 \mathrm{~b}$ & $28.91 \mathrm{~b}$ \\
\hline $\mathrm{C}_{2}$ & 37.25 & $23.00 \mathrm{a}$ & $30.14 \mathrm{a}$ \\
\hline $\mathrm{C}_{3}$ & 36.19 & $23.21 \mathrm{a}$ & $29.70 \mathrm{ab}$ \\
\hline $\operatorname{LSD}_{(0.05)}$ & 2.01 & 1.07 & 1.06 \\
\hline \multicolumn{4}{|c|}{ Nitrogen levels (NL) } \\
\hline $\mathrm{NL}_{1}$ & 35.90 & $21.69 \mathrm{~b}$ & $28.79 \mathrm{~b}$ \\
\hline $\mathrm{NL}_{2}$ & 37.26 & $23.50 \mathrm{a}$ & 30.38 a \\
\hline Significance level & ns & * & $*$ \\
\hline \multicolumn{4}{|l|}{$\begin{array}{l}\mathrm{N} \text { application timings } \\
\text { (NT) }\end{array}$} \\
\hline $\mathrm{NT}_{1}$ & $35.90 \mathrm{~b}$ & 22.93 & 29.44 \\
\hline $\mathrm{NT}_{2}$ & $36.45 \mathrm{ab}$ & 22.57 & 29.76 \\
\hline $\mathrm{NT}_{3}$ & $37.37 \mathrm{a}$ & 22.58 & 29.55 \\
\hline $\operatorname{LSD}_{(0.05)}$ & 1.42 & 0.83 & 0.81 \\
\hline \multicolumn{4}{|l|}{ Planting density (PD) } \\
\hline $\mathrm{PD}_{1}$ & 36.24 & $23.03 \mathrm{a}$ & 29.5 \\
\hline $\mathrm{PD}_{2}$ & 36.92 & $22.16 b$ & 29.66 \\
\hline Significance level & * & * & ns \\
\hline Year $\left(^{*}\right)$ & $22.59 \mathrm{~b}$ & $36.58 \mathrm{a}$ & \\
\hline
\end{tabular}

Note: Cuttings $(C)$ : no-cut $\left(C_{1}\right)$, cutting $\left(C_{2}\right)$, grazing $\left(C_{3}\right)$; Nitrogen levels $(\mathrm{NL}): 80 \mathrm{~kg} \mathrm{ha}^{-1}\left(\mathrm{NL}_{1}\right), 120 \mathrm{~kg} \mathrm{ha}^{-1}\left(\mathrm{NL}_{2}\right)$; Nitrogen timings: Full application at sowing $\left(\mathrm{NT}_{1}\right)$, half dose of $\mathrm{N}$ both at sowing and start of rosette stage $\left(\mathrm{NT}_{2}\right)$, one third dose of $\mathrm{N}$ each at sowing, start of rosette stage and soon after cut at late rosette stage (60 days after sowing $\left(\mathrm{NT}_{3}\right)$; Planting density (PD): 20 plants $\mathrm{m}^{-2}\left(\mathrm{PD}_{1}\right), 40$ plants $\mathrm{m}^{-2}\left(\mathrm{PD}_{2}\right)$. Means of the same category followed by different letters are significantly different at $5 \%$ level of probability using $\operatorname{LSD}_{(0.05)}$ test. ns = non-significant, * = Significant at $5 \%$ level of probability. 


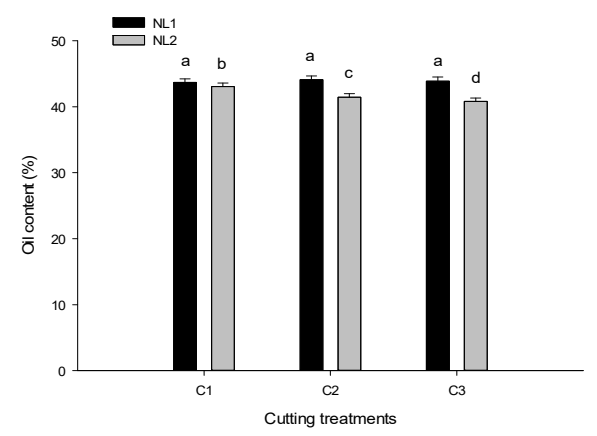

(A)

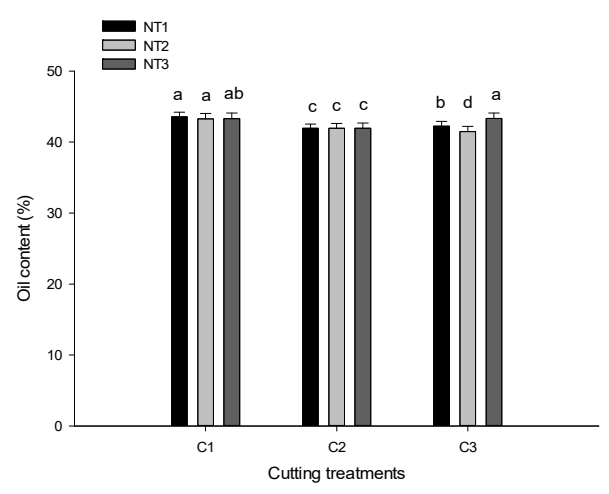

(B)

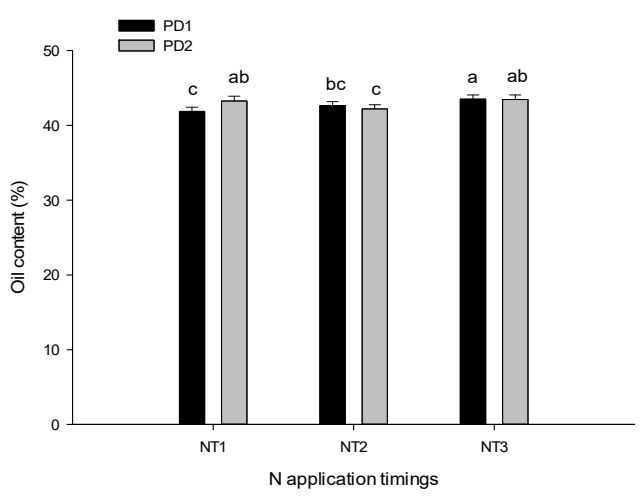

(C)

Figure 2. The interactive effects of nitrogen levels and cutting treatments (A), nitrogen application timings and cutting treatments (B), and nitrogen application timings and planting densities (C) for oil content. Vertical bars represent Standard Error. Note: Cuttings $(C)$ : no-cut $\left(C_{1}\right)$, cutting $\left(C_{2}\right)$, grazing $\left(\mathrm{C}_{3}\right)$; Nitrogen levels (NL): $80 \mathrm{~kg} \mathrm{ha}^{-1}\left(\mathrm{NL}_{1}\right), 120 \mathrm{~kg} \mathrm{ha}^{-1}\left(\mathrm{NL}_{2}\right)$; Nitrogen timings: Full application at sowing $\left(\mathrm{NT}_{1}\right)$, half dose of $\mathrm{N}$ both at sowing and start of rosette stage $\left(\mathrm{NT}_{2}\right)$, one third dose of $\mathrm{N}$ each at sowing, start of rosette stage and soon after cut at late rosette stage (60 days after sowing $\left(\mathrm{NT}_{3}\right)$; Planting density (PD): 20 plants $\mathrm{m}^{-2}\left(\mathrm{PD}_{1}\right), 40$ plants $\mathrm{m}^{-2}\left(\mathrm{PD}_{2}\right)$.

\subsubsection{Erucic acid Content (\%)}

Canola erucic acid content was significantly influenced by $\mathrm{C}$ and NT, while the effect of year, NL and PD was non-significant (Table 7). Erucic acid content was $7.7 \%$ higher in $C_{3}$ than other cutting treatments. Similarly, $\mathrm{NT}_{1}$ had higher erucic acid content than $\mathrm{NT}_{2}$ or $\mathrm{NT}_{3}$. In addition, in the first year the content was $8.07 \%$ higher than 2 nd year of the study. However, significant $C \times P D$ interaction revealed that erucic acid content increased with imposition of $C_{2}$ and $C_{3}$ than $C_{1}$ under $P_{1}$. No or least variation in erucic acid content in all cutting treatments was noted under $\mathrm{PD}_{2}$ treatment (Figure 3 ). 
Table 7. Erucic acid (\%) canola as affected by cutting treatments, nitrogen levels and application timings under varying planting densities.

\begin{tabular}{|c|c|c|c|}
\hline Variables & 2012-2013 & 2013-2014 & Two Years Average \\
\hline \multicolumn{4}{|c|}{ Cutting treatments $(\mathrm{C})$} \\
\hline$C_{1}$ & $32.3 \mathrm{~b}$ & $31.2 \mathrm{~b}$ & $31.0 \mathrm{~b}$ \\
\hline $\mathrm{C}_{2}$ & $34.1 \mathrm{ab}$ & $33.1 \mathrm{ab}$ & $32.9 \mathrm{a}$ \\
\hline $\mathrm{C}_{3}$ & $34.7 \mathrm{a}$ & $33.6 \mathrm{a}$ & $33.4 \mathrm{a}$ \\
\hline $\operatorname{LSD}_{(0.05)}$ & 1.94 & 1.91 & 1.26 \\
\hline \multicolumn{4}{|c|}{ Nitrogen levels (NL) } \\
\hline $\mathrm{NL}_{1}$ & 33.4 & 32.3 & 32.1 \\
\hline $\mathrm{NL}_{2}$ & 34.1 & 32.9 & 32.8 \\
\hline Significance level & ns & ns & ns \\
\hline \multicolumn{4}{|l|}{$\begin{array}{c}\mathrm{N} \text { application timings } \\
\text { (NT) }\end{array}$} \\
\hline $\mathrm{NT}_{1}$ & 33.9 & 32.8 & $32.9 \mathrm{a}$ \\
\hline $\mathrm{NT}_{2}$ & 33.9 & 32.8 & $32.0 \mathrm{~b}$ \\
\hline $\mathrm{NT}_{3}$ & 33.3 & 32.1 & $32.4 \mathrm{ab}$ \\
\hline $\operatorname{LSD}_{(0.05)}$ & ns & ns & 0.805 \\
\hline \multicolumn{4}{|l|}{ Planting density (PD) } \\
\hline $\mathrm{PD}_{1}$ & 33.7 & 32.6 & 32.3 \\
\hline $\mathrm{PD}_{2}$ & 33.7 & 32.5 & 32.6 \\
\hline Significance level & $\mathrm{ns}$ & ns & ns \\
\hline Year $\left(^{*}\right)$ & $33.72 \mathrm{a}$ & $31.2 \mathrm{~b}$ & \\
\hline
\end{tabular}

Note: Cuttings $(C)$ : no-cut $\left(C_{1}\right)$, cutting $\left(C_{2}\right)$, grazing $\left(C_{3}\right)$; Nitrogen levels $(N L) 80 \mathrm{~kg} \mathrm{ha}^{-1}\left(\mathrm{NL}_{1}\right), 120 \mathrm{~kg} \mathrm{ha}^{-1}\left(\mathrm{NL}_{2}\right)$; Nitrogen timings: Full application at sowing $\left(\mathrm{NT}_{1}\right)$, half dose of $\mathrm{N}$ both at sowing and start of rosette stage $\left(\mathrm{NT}_{2}\right)$, one third dose of $\mathrm{N}$ each at sowing, start of rosette stage and soon after cut at late rosette stage (60 days after sowing $\left(\mathrm{NT}_{3}\right)$; Planting density (PD): 20 plants $\mathrm{m}^{-2}\left(\mathrm{PD}_{1}\right), 40$ plants $\mathrm{m}^{-2}\left(\mathrm{PD}_{2}\right)$. Means of the same category followed by different letters are significantly different at $5 \%$ level of probability using $\operatorname{LSD}_{(0.05)}$ test. ns = non-significant, * = Significant at $5 \%$ level of probability.

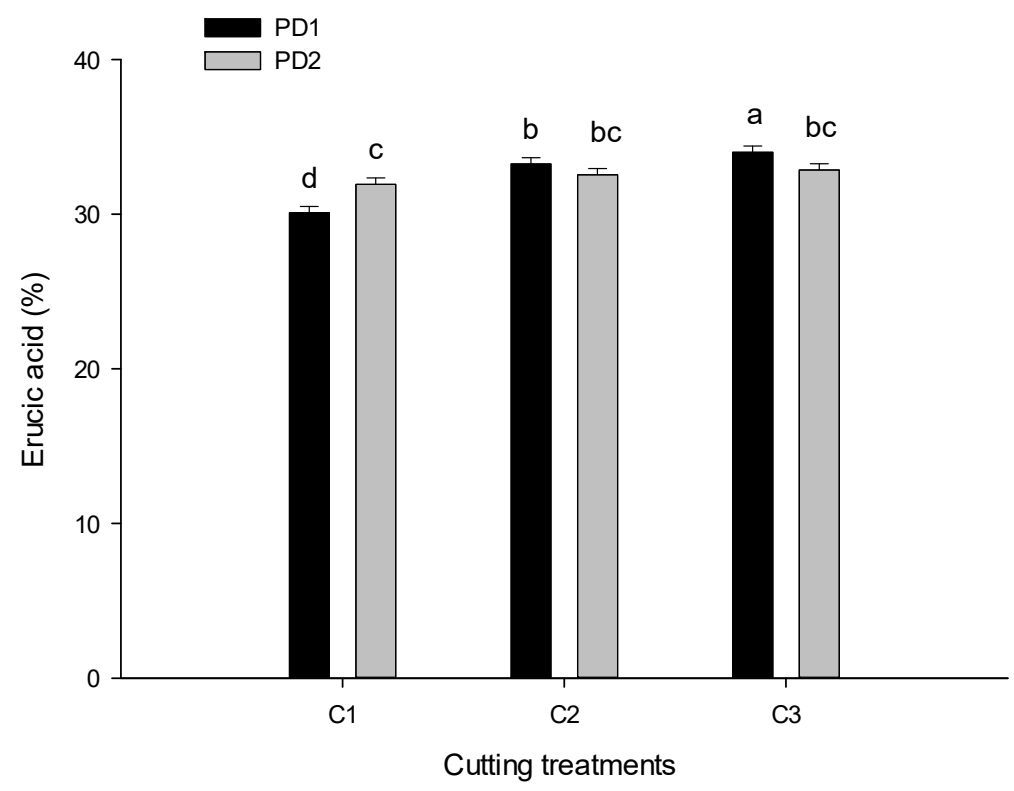

Figure 3. The interactive effect of planting density and cutting treatments of Erucic acid (\%) for canola seed. Vertical bars represent Standard Error. Note: Cuttings $(C)$ : no-cut $\left(C_{1}\right)$, cutting $\left(C_{2}\right)$, grazing $\left(C_{3}\right)$; Planting density (PD): 20 plants $\mathrm{m}^{-2}\left(\mathrm{PD}_{1}\right), 40$ plants $\mathrm{m}^{-2}\left(\mathrm{PD}_{2}\right)$.

\subsubsection{Glucosinolates Concentration}

Canola glucosinolates concentration was substantially impacted by C, NL and year, while the effect of NT and, PD and year was not significant (Table 8). Glucosinolates content was $4 \%$ higher 
in $C_{1}$ followed by $C_{2}$, while $C_{3}$ resulted in lower content of glucosinolates. Similarly, glucosinolates concentration increased with the increased of $\mathrm{N}$ level from 80 to $120 \mathrm{~kg} \mathrm{ha}^{-1}$. Glucosinolates content was $6 \%$ higher in second year compared with compared with first year. The $\mathrm{C} \times \mathrm{NL}$ interaction indicated that increasing $\mathrm{N}$ from 80 upto $120 \mathrm{~kg} \mathrm{ha}^{-1}$ increased the glucosinolates contents in all cutting treatments. However, the glucosinolates were markedly lower in $C_{3}$ and $C_{2}$ than $C_{1}$ under $\mathrm{NL}_{1}$ (Figure 4).

Table 8. Canola glucosinolates content $\left(\mu \mathrm{mol} \mathrm{g}^{-1}\right)$ as affected by cutting treatments, nitrogen levels and application timings under varying planting densities.

\begin{tabular}{|c|c|c|c|}
\hline Variables. & 2012-2013 & 2013-2014 & Two Years Average \\
\hline \multicolumn{4}{|c|}{ Cutting treatments $(C)$} \\
\hline$C_{1}$ & $59.97 \mathrm{~b}$ & $66.18 \mathrm{a}$ & $64.48 \mathrm{a}$ \\
\hline $\mathrm{C}_{2}$ & $62.78 \mathrm{a}$ & $63.67 \mathrm{~b}$ & $61.82 \mathrm{~b}$ \\
\hline $\mathrm{C}_{3}$ & $58.03 \mathrm{c}$ & $62.46 \mathrm{~b}$ & $60.25 c$ \\
\hline $\operatorname{LSD}_{(0.05)}$ & 1.88 & 1.91 & 1.26 \\
\hline \multicolumn{4}{|c|}{ Nitrogen levels (NL) } \\
\hline $\mathrm{NL}_{1}$ & $57.59 \mathrm{~b}$ & $61.42 \mathrm{a}$ & $59.61 \mathrm{~b}$ \\
\hline $\mathrm{NL}_{2}$ & 62.93 a & $66.79 \mathrm{~b}$ & $64.86 \mathrm{a}$ \\
\hline Significance level & $*$ & $*$ & * \\
\hline \multicolumn{4}{|c|}{$\begin{array}{l}\mathrm{N} \text { application timings } \\
\text { (NT) }\end{array}$} \\
\hline $\mathrm{NT}_{1}$ & 60.07 & 63.74 & 62.45 \\
\hline $\mathrm{NT}_{2}$ & 60.27 & 64.26 & 62.23 \\
\hline $\mathrm{NT}_{3}$ & 60.45 & 64.32 & 61.87 \\
\hline $\operatorname{LSD}_{(0.05)}$ & 1.07 & 1.51 & 0.99 \\
\hline \multicolumn{4}{|l|}{ Planting density (PD) } \\
\hline $\mathrm{PD}_{1}$ & 60.76 & 64.29 & 62.11 \\
\hline $\mathrm{PD}_{2}$ & 59.76 & 63.93 & 62.26 \\
\hline Significance level & ns & ns & ns \\
\hline Year $\left(^{*}\right)$ & $60.26 \mathrm{~b}$ & $64.11 \mathrm{a}$ & \\
\hline
\end{tabular}

Note: Cuttings $(\mathrm{C})$ : no-cut $\left(\mathrm{C}_{1}\right)$, cutting $\left(\mathrm{C}_{2}\right)$, grazing $\left(\mathrm{C}_{3}\right)$; Nitrogen levels $(\mathrm{NL}) 80 \mathrm{~kg} \mathrm{ha}^{-1}\left(\mathrm{NL}_{1}\right), 120 \mathrm{~kg} \mathrm{ha}^{-1}\left(\mathrm{NL}_{2}\right)$; Nitrogen timings: Full application at sowing $\left(\mathrm{NT}_{1}\right)$, half dose of $\mathrm{N}$ both at sowing and start of rosette stage $\left(\mathrm{NT}_{2}\right)$, one third dose of $\mathrm{N}$ each at sowing, start of rosette stage and soon after cut at late rosette stage (60 days after sowing $\left(\mathrm{NT}_{3}\right)$; Planting density (PD): 20 plants $\mathrm{m}^{-2}\left(\mathrm{PD}_{1}\right), 40$ plants $\mathrm{m}^{-2}\left(\mathrm{PD}_{2}\right)$. Means of the same category followed by different letters are significantly different at $5 \%$ level of probability using $\mathrm{LSD}_{(0.05)}$ test. ns = non-significant, * = Significant at $5 \%$ level of probability.

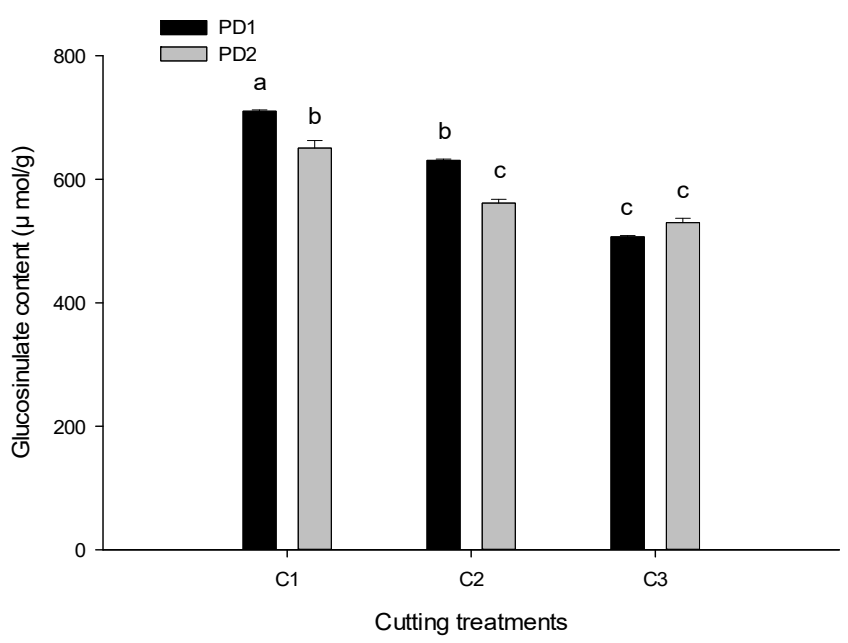

Figure 4. Interaction between cutting treatments and nitrogen levels for glucosinulates content. Vertical bars represent Standard Error. Note: Cuttings $(C)$ : no-cut $\left(C_{1}\right)$, cutting $\left(C_{2}\right)$, grazing $\left(C_{3}\right)$; Planting density (PD): 20 plants $\mathrm{m}^{-2}\left(\mathrm{PD}_{1}\right), 40$ plants $\mathrm{m}^{-2}\left(\mathrm{PD}_{2}\right)$. 


\subsection{Economic Analysis}

Economic analysis showed that $C_{2}$ produced higher net income (USD 1202.1) compared to that of $C_{1}$ (USD 1057.2) (Table 9). However, in $C_{1}$ plots net income was higher compared to $C_{3}$ (USD 121). $C_{2}$ and $C_{3}$ plots reduced grain yield by $15 \%$ and $23 \%$, respectively, than $C_{1}$ plots. Likewise, value cost ratio (VCR) of $C_{2}$ was higher compared with $C_{3}$ and $C_{1}$ plots. Furthermore, higher VCR (4.16) was found in $\mathrm{C}_{2}$ where $\mathrm{N}$ at the rate of $80 \mathrm{~kg} \mathrm{ha}^{-1}$ in $\mathrm{NT}_{2}$ with high density (40 plants $\mathrm{m}^{-2}$ ) was followed by $\mathrm{C}_{2}$ and received at $\mathrm{NL}_{1}$ with $\mathrm{PD}_{2}$. The lower VCR (1.58) in $\mathrm{C}_{3}$ received at $\mathrm{NL}_{2}$ in $\mathrm{NT}_{3}$ in $\mathrm{PD}_{1}$ (Table 10). The net income from fodder of canola in $\mathrm{C}_{2}$ plots were recorded on the basis of area as per usual practice at the same farm.

Table 9. Economic analysis of dual-purpose canola.

\begin{tabular}{cccc}
\hline Yield, Value or Cost & $\mathbf{C}_{\mathbf{1}}$ & $\mathbf{C}_{\mathbf{2}}$ & $\mathbf{C}_{\mathbf{3}}$ \\
\hline Forage yield $\left(\mathrm{kg} \mathrm{ha}^{-1}\right)$ & 0.0 & 13.9 & 14.1 \\
Grain yield $\left(\mathrm{kg} \mathrm{ha}^{-1}\right)$ & 12.4 & 10.5 & 9.5 \\
Straw yield $\left(\mathrm{kg} \mathrm{ha}^{-1}\right)$ & 92.1 & 74.3 & 63.8 \\
Forage value (USD) & 0.0 & 69.5 & 70.3 \\
Grain value (USD) & 884.5 & 1067.1 & 863.4 \\
Straw value (USD) & 552.4 & 446.1 & 383.1 \\
Gross income (USD) & 1436.9 & 1582.8 & 1316.9 \\
Net income over control (USD) & 1057.2 & 1202.1 & 936.2 \\
Value cost ratio (VCR \%) & 2.78 & 3.1 & 2.4 \\
\hline
\end{tabular}

Note: Cuttings $(C)$ : no-cut $\left(C_{1}\right)$, cutting $\left(C_{2}\right)$, grazing $\left(C_{3}\right)$.

Table 10. The interactive effects of different factors over economic benefits (VCR values).

\begin{tabular}{cccccc}
\hline Plant density $\mathbf{~ m}^{-\mathbf{2}}$ & Cutting & $\mathbf{N}$ rate & $\mathbf{N T}_{\mathbf{1}}$ & $\mathbf{N T}_{\mathbf{2}}$ & $\mathbf{N T}_{\mathbf{3}}$ \\
\hline $\mathrm{PD}_{1}$ & $\mathrm{C}_{1}$ & $\mathrm{NL}_{1}$ & 2.95 & 2.57 & 2.82 \\
& $\mathrm{C}_{1}$ & $\mathrm{NL}_{2}$ & 2.59 & 2.21 & 2.71 \\
& $\mathrm{C}_{2}$ & $\mathrm{NL}_{1}$ & 3.00 & 3.35 & 3.00 \\
& $\mathrm{C}_{2}$ & $\mathrm{NL}_{2}$ & 2.71 & 3.36 & 3.17 \\
& $\mathrm{C}_{3}$ & $\mathrm{NL}_{1}$ & 3.09 & 3.35 & 2.92 \\
$\mathrm{CD}_{3}$ & $\mathrm{NL}_{2}$ & 2.85 & 2.22 & 1.58 \\
& $\mathrm{CL}_{1}$ & 2.60 & 3.30 & 3.10 \\
& $\mathrm{NL}_{1}$ & $\mathrm{NL}_{2}$ & 3.02 & 2.69 & 2.80 \\
& $\mathrm{CL}_{1}$ & 3.52 & 4.16 & 2.57 \\
& $\mathrm{C}_{2}$ & $\mathrm{NL}_{2}$ & 3.22 & 3.06 & 2.83 \\
& $\mathrm{C}_{2}$ & $\mathrm{NL}_{1}$ & 2.18 & 2.63 & 2.07 \\
& $\mathrm{C}_{3}$ & $\mathrm{NL}_{2}$ & 2.59 & 2.23 & 1.96 \\
\hline
\end{tabular}

Note: Cuttings $(C)$ : no-cut $\left(\mathrm{C}_{1}\right)$, cutting $\left(\mathrm{C}_{2}\right)$, grazing $\left(\mathrm{C}_{3}\right)$; Nitrogen levels $(\mathrm{NL}) 80 \mathrm{~kg} \mathrm{ha}^{-1}\left(\mathrm{NL}_{1}\right), 120 \mathrm{~kg} \mathrm{ha}^{-1}\left(\mathrm{NL}_{2}\right)$; Nitrogen timings: Full application at sowing $\left(\mathrm{NT}_{1}\right)$, half dose of $\mathrm{N}$ both at sowing and start of rosette stage $\left(\mathrm{NT}_{2}\right)$, one third dose of $\mathrm{N}$ each at sowing, start of rosette stage and soon after cut at late rosette stage (60 days after sowing $\left(\mathrm{NT}_{3}\right)$; Planting density (PD): 20 plants $\mathrm{m}^{-2}\left(\mathrm{PD}_{1}\right), 40$ plants $\mathrm{m}^{-2}\left(\mathrm{PD}_{2}\right)$.

\section{Discussion}

\subsection{Crop Yield}

\subsubsection{Effects of Grazing and Cut on Canola Yield}

Biological yield is the function of increase rate and growth duration both of which indicate the possibility for improved yield. In this study, biological yield was higher in no-cut plots followed by cut and grazed plots. Biological yield recovered rapidly in cut and grazed plots but the removal of branches in initial grazing and cuttings had led to the differences among the means. Delay in flowering may affect biological yield, when grazing removed the main auxiliary buds from the stems [15]. In our study biological yield was higher in second year (2013-2014) compared with first year (2012-2013), 
might be due to that in the second year, canola was sown for 15 days earlier than first year. Earlier sown plans of dual-purpose cropping for better results [16]. Reduction in biological yield was associated with the removal of branches in flowering, and not affected on seed yield [17]. Gross marginal value of DP canola is greater than grains only. In most cases grazing during early growth stages does not show significant results in the reduction of seed yield [18,19], while grazing after vegetative stage caused reduction up to $25 \%$ or even more $[15,16]$. Cutting treatments caused significant reduction in grain yield, might be due to less re-growth ability of plants in cut and grazed plots, and they were unable to regenerate quickly and reach to the growth of plants of no cut plots. The possible reason for this substantial decrease in yield of grazed and cut plots might be the removal of main stem either manually or by sheep grazing.

\subsubsection{Effects of $\mathrm{N}$ fertilizer, Application Timings on Canola Yield}

Canola crop responded well to $\mathrm{N}$ application timings. In these experiments, $3 \%$ and $1 \%$ increase in biological yield of canola was recorded in plots where $\mathrm{N}$ was applied in two or three splits as compared with sole application, respectively. The increase in yield with split application of $\mathrm{N}$ at seedling, rosette stage and early flowering [20]. Similarly, application of $\mathrm{N}$ at the rate of $100 \mathrm{~kg} \mathrm{ha}^{-1}$ in split form (half each at sowing and soon after grazing) increased the biological yield up to $3.95 \mathrm{tha}^{-1}$ [21], and nitrogen in split form resulted better than sole application [22].

Canola crop requires high amount of $\mathrm{N}$ fertilizer compared to cereals to produce high yields [23]. In our study, seed yield increased by $6 \%$ when $\mathrm{N}$ rate mounted from 80 to $120 \mathrm{~kg} \mathrm{ha}^{-1}$. Higher yields of canola achieved in the current study also highlights the high levels of $\mathrm{N}$ fertilizer which must be applied to achieve enhanced seed yield. In general, for canola crop $80 \mathrm{~kg} \mathrm{~N} \mathrm{ha}^{-1}$ may be applied in the growing season for each $1 \mathrm{t} /$ ha predictable yield [21]. Several studies observed no significant results with further $\left(200 \mathrm{~kg} \mathrm{ha}^{-1}\right)$ increase in $\mathrm{N}$ levels $[3,24]$. While, in our study, the improvement in grain yield can be attributed to $\mathrm{N}$ fertilizer at $120 \mathrm{~kg} \mathrm{ha}^{-1}$ in two splits half at sowing while remaining at rosette stage. The split application of $\mathrm{N}$ fertilizer provides flexibility in their fertilizer program, and attracts farmers. Further, higher grain yield was noted in plots where $\mathrm{N}$ was applied in two splits as compared with single fertilization of $\mathrm{N}$. The splits application of $\mathrm{N}$ fertilizer benefited crop growth and ensure availability of nutrients at two splits, one at sowing and second at rosette stage which may result in higher grain yield of faba bean $[25,26]$.

\subsubsection{Effects of Planting Densities on Canola Yield}

Optimum planting is important to attain high yield and is a best option for reducing lodging among the plants. Biological yield increased by $3.3 \%$ with planting density of 40 plants $\mathrm{m}^{-2}$ compared to 20 plants $\mathrm{m}^{-2}$. This difference may be mainly due to increase in plants per unit area. Dahmardeh et al. compared three planting densities $\left(12.5,16.7\right.$, and 20 plants $\left.\mathrm{m}^{-2}\right)$ and found that biological yield of canola was highest for 20 plant $\mathrm{m}^{-2}$ compared to other planting density [27]. The inter-competition for nutrients among the plants might be a reason for the lower biological yield. Planting density is an important factor which determines the yield and which is individually affected by the climatic conditions and production system of an area as well [28]. In our study, planting density had non-significant effects on grain yield which indicated that 20 and 40 plants $\mathrm{m}^{-2}$ gave same results for grain yield of canola. However, higher yield in least densities indicated the proper utilization and maximum facilitation of nutrients [3].

\subsection{Qualitative Traits of Canola}

\subsubsection{Effects of Grazing and Cut on Quality Traits of Canola}

The improvement in quality of seed is the primary objective of breeding oil seed crops to fulfill upcoming edible oil requirements [29]. Oil content is mainly related with genetics for most of the species and varieties but the role of environment cannot be ignored. Cutting and grazing declined 
oil content of canola. We do not agree with the findings of Kirkegaard et al. that allowing sheep for grazing before bud elongation had no impact on oil content of canola seed [16]. Protein content in cut plots was higher than no cut and grazed plots while, glucosinolates was maximum in no cut plots compared to grazed and cut plots. However, year had significant effect on oil content of canola. Almost $113.6 \%$ higher oil content was recorded in second year compared to that of first year. Likewise, in second year protein content was increased up to $161.9 \%$ as compared to first year.

\subsubsection{Effects of N Fertilizer, Application Timings on Quality Traits of Canola}

The $\mathrm{N}$ fertilizer had a negative correlation with oil content of the seeds [30,31]. Increasing $\mathrm{N}$ level from 80 to $120 \mathrm{~kg} \mathrm{ha}^{-1}$ decreased the oil content of canola seed. Likewise, higher oil content was measured in plots where $\mathrm{N}$ was applied in splits compared with sole $\mathrm{N}$ application. Further, seed oil content of canola reduced significantly with increase in $\mathrm{N}$ levels from 0 to $200 \mathrm{~kg} \mathrm{ha}^{-1}$ [32,33], and the highest oil content $(43.08 \%)$ in plots with low $\mathrm{N}$ rates $\left(50 \mathrm{~kg} \mathrm{ha}^{-1}\right)$ while lowest oil content $(38.64 \%)$ was recorded with high levels of $\mathrm{N}$ fertilizer $\left(200 \mathrm{~kg} \mathrm{ha}^{-1}\right)$ [34]. However, the reduction in oil content due to increase in $\mathrm{N}$ levels [31,34]. For example, the accessibility of sugar for oil synthesis becomes less with increase in $\mathrm{N}$ rates, that the application of high rates of nitrogen fertilizer increased the amount of $\mathrm{N}$ containing protein; so this protein development goes through a competition for photosynthesis, as a consequence of less amount of the later is obtainable for fats production $[32,35,36]$. This inverse relationship between oil and protein content with increase in $\mathrm{N}$ levels may also be the possible reason [37]. However, our data did not agree with the findings of Brennan et al. (2000) who concluded that oil content is not going to decrease with increased in $\mathrm{N}$ rates [38]. It is also noticeable that protein content of canola seed improved with rising levels of nitrogen.

Nitrogen is the integral part of protein structures and involved in many other plant metabolic processes. Thus, increasing $\mathrm{N}$ levels increased the protein content of canola seeds. $\mathrm{N}$ at $120 \mathrm{~kg} \mathrm{~N} \mathrm{ha}^{-1}$ application had higher protein content over $80 \mathrm{~kg} \mathrm{ha}^{-1}$. The higher protein value is the evidence of negative correlation between oil content and protein content. Both these are inversely proportional to each other [6]. Increased N supply helps in increasing protein synthesis without compromising oil content reduction [18]. Similarly, split application resulted in higher protein contents compared with sole $\mathrm{N}$ application. The protein content of canola increased from $22.7 \%$ to $23.7 \%$ with the increasing $\mathrm{N}$ rates from 80 to $160 \mathrm{~kg} \mathrm{ha}^{-1}$ [39]. Glucosinolates contents were significantly affected by $\mathrm{N}$ levels but $\mathrm{N}$ application timing had a non-significant effect on glucosinolates contents. Glucosinolates contents increased from 59 to $64 \mu \mathrm{mol} \mathrm{g}-1$ with increasing $\mathrm{N}$ from 80 to $120 \mathrm{~kg} \mathrm{ha}^{-1}$. These data indicated that increasing $\mathrm{N}$ levels significantly increased glucosinolates contents. The increase in glucosinolates contents due to $\mathrm{N}$ fertilization was also reported by [35,40-42]. Glucosinolates structure contains $\mathrm{N}$ therefore high $\mathrm{N}$ concentration may be influenced by the addition of $\mathrm{N}$ fertilizers [43].

\subsubsection{Effects of Planting Densities on Quality Traits of Canola}

Competition among the plants due to high planting density can result in poor quality attributes, impairs plant growth, reduced biomass formation, and consequently yield loss due to low nutrients uptake, and disruption in leaf structural and functional characteristics [44-46]. Increasing density decreases oil content may be due to inter plant competition for nutrients. Plants grown at 40 plants $\mathrm{m}^{-2}$ had higher oil content compared to 20 plants $\mathrm{m}^{-2}$. In contrary, no significant variation in oil content with increase in planting densities from 45 to 80 plants $\mathrm{m}^{-2}$ [47].

\subsection{Interaction Effect of Factors}

The proper nutrients at proper time to any crop is important [6], and canola crop requires a higher amount of nutrients, and available nitrogen $(\mathrm{N})$ compared with that of cereals [7]. Split application of $\mathrm{N}$ fertilizer has become more popular in terms of high nitrogen use efficiency. Therefore, an appropriate rate and timing of $\mathrm{N}$ fertilizer application is one of the most important aspects of successful canola 
production [3]. These findings support our results that the interactive effects of NL2 with NT2 at PD1 were more qualitative and productive for canola under dual purpose.

\subsection{Economics Benefits}

The effectiveness of dual-purpose canola can be predictable by the economic analysis of no-cut, cut and grazing systems. Cut plots produced higher net income as compared to no-cut and grazed plots. The higher net income and thus higher value cost ratio (VCR) value of cut plots was due to high fodder and grains as compared to no-cut system. However, grazed plots reduced VCR value by $10 \%$ and net income by $69 \%$ as compared to no-cut plots. Our results are against with the findings that higher net income ( $\$ 240$ to $\$ 500$ ) for grazed plots as compared to no-cut or grains only system [47].

\section{Conclusions}

The study revealed that integration of DP cropping would increase farm productivity, profitability and flexibility of the farm operations. It is an innovation which captures more food by increasing crop and livestock production on the same farm. $\mathrm{C}_{2}$ caused a $15 \%$ reduction in grain yield of canola; however, it fetched additional income of USD 143.6 compared to $C_{1}$. In case of $C_{3}, 23 \%$ reduction was resulted in grain yield of canola with income of USD 117.7 from fodder yield of the same canola. Treatment $\mathrm{NL}_{2}$ produced higher seed yields and improved quality traits of canola compared with $\mathrm{NL}_{1}$. Further, $\mathrm{NL}_{2}$ increased grain yield and qualitative parameters of canola. Crops under $\mathrm{PD}_{2}$ produced more biological yield compared to $\mathrm{PD}_{1}$. However, seed yield was higher at $\mathrm{PD}_{1}$. Dual purpose cropping is a classical way which can contribute to continued development of sustainable agriculture systems. Currently, Pakistan is facing a serious shortage of edible oils and food insecurity threats. Therefore, the use of application of $\mathrm{N}$ in two splits at $120 \mathrm{~kg} \mathrm{~N}^{-1}$ coupled with 20 plants $\mathrm{m}^{-2}$ is a good option to achieve better qualitative attributes and high yield of canola under dual purpose system.

Author Contributions: S.J., performed experiment, K.A., F.S., and M.J., help in data analysis and making figures; M.A., supervision; A.K. (Ahmad Khan), and A.Z., S.A., A.K. (Aziz Khan), Methodology and software; S.B., and F.M., worked as investigators; S.J., write final full length draft; M.M.A., F.J., N.A., and M.A.K., revised and edit English language; F.W., revised article and provide funding; S.Z., Data curation; Writing-Originial draft; N.U.A., Writing - Review \& editing. All authors have read and agreed to the published version of the manuscript.

Funding: This review is financially supported by the Guangxi Innovation-Driven Development Project (GuiKe AA18242040, Guangxi Natural Science Foundation (2018GXNSFBA294016).

Conflicts of Interest: The authors declare no conflict of interest.

\section{References}

1. Barlog, P.; Grzebisz, W. Effect of timing and nitrogen fertilizer application on winter oilseed rape (Brassica napus L.). I. Growth Dynamics and Seed Yield. J. Agron. Crop. Sci. 2004, 190, 305-310. [CrossRef]

2. GOP. Economic Survey Report, 2003-2004; Govt. of Pakistan, Finance Division: Islamabad, Pakistan, 2007; pp. 3-4.

3. Cheema, M.A.; Malik, M.A.; Hussain, A.; Shah, S.H.; Basra, S.M.A. Effects of time and rate of nitrogen and phosphorus application on growth and seed and oil yields of canola. J. Agron. Crop. Sci. 2001, 186, 103-110. [CrossRef]

4. Grombacher, A.; Nelson, L. Canola Production. A University of Nebraska Neb Guide Publication, G92-1076-A; Institute of Agriculture and Natural Resources, University of Nebraska-Lincoln: Lincoln, NE, USA, 1992.

5. Gunasekera, C.P.; Martin, L.D.; Siddique, K.H.M.; Walton, G.H. Genotype by environment interactions of indian mustard (Brassica juncea L.) and canola (Brassica napus L.) in mediterranean-type environments: Ii. Oil and protein concentrations in seed. Eur. J. Agron. 2006, 25, 13-21. [CrossRef]

6. Hao, X.C.; Chang, C.; Travis, G.J. Short communication: Effect of long-term cattle manure application on relations between nitrogen and oil content in canola seed. J. Plant Nutr. Soil Sci. 2004, 167, 214-215. [CrossRef] 
7. Hocking, P.J.; Strapper, M. Effect of sowing time and nitrogen fertilizer on canola and wheat, and nitrogen fertilizer on Indian mustard. II. Nitrogen concentrations, $\mathrm{N}$ accumulation, and $\mathrm{N}$ use efficiency. Aust. J. Agric. Res. 2001, 52, 635-644. [CrossRef]

8. Faraji, A.; Sadeghi, S.; Asadi, M. Evaluation of nitrogen and irrigationeffects on yield and yield components of canola varieties in Gonbad. J. Agric. Res. Nat. Resour. 2005, 12, 63-72.

9. Wright, G.C.; Smith, C.J.; Woodroffe, M.R. The effect of irrigation and nitrogen fertilizer on rapeseed (Brassica napus) production in South- Eastern Australia, I. Growth and seed yield. Irrig. Sci. 1988, 9, 1-13. [CrossRef]

10. Sommer, S.G.; Schjoerring, J.K.; Denmead, O.T. Ammonia emission from mineral fertilizers and fertilized crops. Adv. Agron. 2004, 82, 557-622.

11. Kirkegaard, J.A.; Sprague, S.J.; Hamblin, P.J.; Graham, J.M.; Lilley, J.M. Refining crop and livestock management for dual-purpose spring canola (Brassicanapus). Crop Pasture Sci. 2006, 63, 429-443. [CrossRef]

12. Daun, J.K.; Clear, K.M.; Williams, P. Comparison of three whole seed near infrared analyzers for measuring quality components of canola seed. J. Am. Oil Chem. Soc. 1994, 71, 1063-1068. [CrossRef]

13. Canola Council of Canada. The Grower's Manual (Online); Canola Council of Canada: Winnipeg, MB, Canada, 2005.

14. Jan, M.; Shah, P.; Hollington, P.; Khan, M.; Sohail, Q. Agriculture research: Design and analysis, a monograph. NWFP Agric. Univ. Pesh. Pak. 2009, 1, 232-250.

15. Kirkegaard, J.; Sprague, S.J.; Marcroft, S.; Potter, T.D.; Graham, J.; Virgona, J. Identifying canola varieties for dual-purpose use. In Global Issues_Paddock Action, Proceedings of the 14th Australian Agronomy Conference, Adelaide South, Australia, 21-25 September 2008; Australian Society of Agronomy: Gosford, Australia, 2008.

16. Khan, A.H.; Khalil, I.A.; Shah, H. Nutritional yield and oil quality of canola cultivars grown in NWFP. Sarhad J. Agric. 2004, 20, 287-290.

17. Susko, D.J.; Superfisky, B. A comparison of artificial defoliation techniques using canola (Brassica napus). Plant Ecol. 2009, 202, 169-175. [CrossRef]

18. McCormick, J.I.; Virgona, J.M.; Kirkegaard, J.A. Growth and yield of dual-purpose canola (Brassica napus) under drier inland seasonal conditions of south-eastern Australia. Crop Pasture Sci. 2012, 63, 635-646. [CrossRef]

19. Salehian, H.; Rafiey, M.; Fathiand, G.; Siadat, S.A. Effect of plant density on growth and seed yield of colza varieties under Andimeshk conditions. In Proceedings of the 7th Iranian Crop Sciences Congress, Karaj, Iran, 15 March 2007.

20. Sprague, S.J.; Kirkegaard, J.A.; Graham, J.M.; Dove, H.; Kelman, W.M. Crop and livestock production for dual-purpose winter canola (Brassica napus) in the high-rainfall zone of south-eastern Australia. Field Crop. Res. 2014, 156, 30-39. [CrossRef]

21. Al-Jaloud, A.A.; Hussain, G.; Karimulla, S.; Alhamdi, A.H. Effect of irrigation and nitrogen on yield and yield components of two rapeseed cultivars. Agric. Water Manag. 1996, 30, 57-68. [CrossRef]

22. Balint, T.; Rengel, Z.; Allen, D. Australia canola germplasm differs in nitrogen and sulphur defficiency. Aust. J. Agric. Res. 2008, 59, 1164-1174. [CrossRef]

23. Buttar, G.S.; Thind, H.S.; Aujla, M.S. Methods of planting and irrigation at various levels of nitrogen affect the seed yield and water use efficiency in transplanted oil seed rape. Agric. Water Manag. 2005, 85, 253-260. [CrossRef]

24. Cheema, M.A. Production Efficiency of Canola (Brassica napus L.) cv. Shiralee under Different Agro-Management Practices. Ph.D. Thesis, Department of Agronomy, University of Agriculture, Faisalabad, Pakistan, 1999.

25. Zaman, M. Effect of Rate and Time of Nitrogen Application on Growth, Seed Yield and Oil Contents of Canola (Brassica napus L.). Bachelor's Thesis, Department of Agronomy, University of Agriculture, Faisalabad, Pakistan, 2003.

26. Dahmardeh, M.; Mahmood, R.; Valizadeh, J. Effect of plant density and cultivars on growth, yield and yield components of faba bean (Viciafaba L.). Afr. J. Biotechnol. 2010, 9, 8643-8647.

27. Shengwu, H.; Ovesna, J.; Kueera, L.; Kueera, V.; Vyvadilova, M. Evaluation of genetic diversity of Brassica napusgermplasm from China and Europe assessed by RAPD markers. Plant Soil Environ. 2003, 49, 106-113.

28. Kirkegaard, J.A.; Sprague, S.J.; Lilley, J.M.; McCormick, J.I.; Virgona, J.M.; Morrison, M.J. Physiological response of spring canola (Brassica napus) to defoliation in diverse environments. Field Crop. Res. 2012, 125, 61-68. [CrossRef] 
29. Söchtling, H.P.; Verret, J.A. Effects of different cultivation systems (soil management and nitrogen fertilization) on the epidemics of fungal diseases in oilseed rape (Brassica napus L. var. napus). J. Plant Dis. Prot. 2004, 111, 1-29. [CrossRef]

30. Rathke, G.W.; Christen, O.; Diepenbrock, W. Effects of nitrogen source and rate on productivity and quality of winter oilseed rape (Brassica napus L.) grown in different crop rotations. Field Crop. Res. 2005, 94, 103-113. [CrossRef]

31. Akhtar, K.; Wang, W.; Khan, A.; Ren, G.; Afridi, M.Z.; Feng, Y.; Yang, G. Wheat straw mulching offset soil moisture deficient for improving physiological and growth performance of summer sown soybean. Agric. Water Manag. 2019, 211, 16-25. [CrossRef]

32. Saleem, M.; Cheema, A.M.; Malik, A.M. Agro-EconomicAssessment of Canola Planted under Different Levels of Nitrogenand Row Spacing. Int. J. Agric. Biol. 2001, 3, 27-30.

33. Asare, E.; Scarisbrick, D.H. Rate of nitrogen and sulphur fertilizers on yield, yield components and seed quality of oilseed rape (Brassica napus L.). Field Crop. Res. 1995, 44, 41-46. [CrossRef]

34. Omirou, M.D.; Papadopoulou, K.K.; Papastylianou, I.; Constantinou, M.; Karpouzas, D.G.; Asimakopoulos, I.; Ehaliotis, C. Impact on nitrogen and sulfur fertilization on the composition of glucosinolates in relation to sulfur assimilation in different plant organs of broccoli. J. Agric. Food Chem. 2009, 57, 9408-9417. [CrossRef]

35. Holmes, M.R.J. Nitrogen. In Nutrition of the Oilseed Rape Crop; Applied Science Publishers: Barking, UK, 1980; pp. 21-67.

36. Kutcher, H.R.; Malhi, S.S.; Gill, K.S. Topography and management of nitrogen and fungicide affects diseases and productivity of canola. Agron. J. 2005, 97, 533-541. [CrossRef]

37. Brennan, R.F.; Mason, M.G.; Walton, G.H. Effect of nitrogen fertilizer on the concentrations of oil and protein in canola (Brassica napus) seed. J. Plant Nutr. 2000, 23, 339-348. [CrossRef]

38. Ahmad, G.; Jan, A.; Arif, M.; Jan, M.T.; Khattak, R.A. Influence of nitrogen and sulfur fertilization on quality of canola (Brassica napus L.) under rain fed conditions. J. Zhejiang Univ. Sci. B 2007, 8, 731-737. [CrossRef]

39. Narits, L. Effect of nitrogen rate and application time to yield and quality of winter oilseed rape (Brassica napus L. var. oleiferasubvar. biennis). Agron. Res. 2010, 8, 671-686.

40. Chen, X.J.; Zhu, Z.J.; Ni, X.L.; Qian, Q.Q. Effect of Nitrogen and Sulfur Supply on Glucosinolates in Brassica campestris ssp. chiensis. Agric. Sci. China 2006, 5, 603-608. [CrossRef]

41. Zhao, F.; Evans, E.J.; Bilsborrow, P.E.; Syers, J.K. Influence of nitrogen and sulphur on the glucosinolates profile of rapeseed (Brassica napus L.). J. Sci. Food Agric. 2006, 64, 295-304. [CrossRef]

42. Falk, K.L.; Tokuhisa, J.G.; Gershenzon, J. The effect of sulfur nutrition on plant glucosinolates content: Physiology and molecular mechanisms. Plant Biol. 2007, 9, 573-581. [CrossRef]

43. Saeedi, F.H.; Bagheri, H.; Rasoli, S.A.; Ghasemi, J. The effect of planting density on morphological traits of spring canola cultivars in Chaloos region of Iran. Int. J. Agron. Plant Prod. 2013, 4, 911-914.

44. Khan, A.; Zheng, J.; Tan, D.K.Y.; Khan, A.; Akhtar, K.; Kong, X.; Fahad, S. Changes in Leaf Structural and Functional Characteristics when Changing Planting Density at Different Growth Stages Alters Cotton Lint Yield under a New Planting Model. Agronomy 2019, 9, 859. [CrossRef]

45. Khan, A.; Kong, X.; Najeeb, U.; Zheng, J.; Tan, D.K.Y.; Akhtar, K.; Zhou, R. Planting density induced changes in cotton biomass yield, fiber quality, and phosphorus distribution under beta growth model. Agronomy 2019, 9, 500. [CrossRef]

46. Khan, A.; Wang, L.; Ali, S.; Tung, S.A.; Hafeez, A.; Yang, G. Optimal planting density and sowing date can improve cotton yield by maintaining reproductive organ biomass and enhancing potassium uptake. Field Crop. Res. 2017, 214, 164-174. [CrossRef]

47. Gan, Y.; Malhi, S.S.; Brandt, S.; Katepa-Mupondwa, F.; Stevenson, C. Nitrogen use efficiency and nitrogen uptake of juncea Canola under diverse environments. Agron. J. 2007, 100, 285-295. [CrossRef]

(C) 2020 by the authors. Licensee MDPI, Basel, Switzerland. This article is an open access article distributed under the terms and conditions of the Creative Commons Attribution (CC BY) license (http://creativecommons.org/licenses/by/4.0/). 